\title{
Refractory Chronic Lymphocytic Leukemia with Central Nervous System Involvement: A Case Report with Literature Review
}

This article was published in the following Dove Press journal:

Journal of Blood Medicine

\section{Takahisa Nakanishi* \\ Tomoki Ito (D* \\ Shinya Fujita \\ Atsushi Satake (D) \\ Akiko Konishi (D) \\ Masaaki Hotta \\ Hideaki Yoshimura \\ Shosaku Nomura}

First Department of Internal Medicine, Kansai Medical University, Hirakata, Osaka, Japan

*These authors contributed equally to this work
Correspondence: Tomoki Ito First Department of Internal Medicine, Kansai Medical University, 2-5-I Shinmachi, Hirakata, Osaka 573-1010, Japan

Tel $+81-72-804-2425$

Fax +8I-72-804-2506

Email itot@hirakata.kmu.ac.jp

\begin{abstract}
There have been few reports on central nervous system (CNS) involvement in chronic lymphocytic leukemia (CLL). This is an extremely rare disease with poor prognosis, owing to resistance to various treatments. We describe a 33-year-old man with intractable CLL with CNS involvement. He was diagnosed with CLL, with diplopia as the first manifestation. Magnetic resonance imaging revealed a contrast-enhancing tumor in the right temporal lobe, which was diagnosed as CNS involvement in CLL on brain biopsy. High-dose methotrexate therapy was ineffective for this lesion, which was also resistant to subsequent whole-brain irradiation, treatment with fludarabine-cyclophosphamide-rituximab chemoimmunotherapy, and ibrutinib administration. Because no standard protocol exists for CLL with CNS involvement, it is important to accumulate case data to verify the choice of new drugs for administration at an early stage. Therefore, we also conducted a literature review of 50 case reports of CNS lesions in the last 10 years to consider the pathophysiology, diagnosis, and treatment of CNS involvement in CLL. The possibility of new therapeutic agents, eg, ibrutinib and venetoclax, or a combination of these agents and methotrexate, can be envisioned as a treatment strategy for CLL with CNS involvement.
\end{abstract}

Keywords: chronic lymphocytic leukemia, central nervous system involvement, literature review

\section{Introduction}

Chronic lymphocytic leukemia (CLL) is the most frequent adult leukemia in the US and Europe, but is a rare disease in Japan, with a frequency $10 \%$ that in theUS. ${ }^{1}$ The disease typically occurs in older patients, and the median age at diagnosis is 72 years. ${ }^{2}$ Generally, CLL progresses slowly, but some cases progress rapidly and aggressively. ${ }^{3}$ Furthermore, CLL has a highly variable clinical course, and neurological complications arising from direct leukemic involvement in the central nervous system (CNS) are reported in only $1 \%$ of patients with CLL. ${ }^{4,5}$ Here, we present a rare case of a young CLL patient with CNS involvement that was resistant to various therapies. CLL treatment has improved considerably in the last decade; however, it remains unclear which the best treatment for CNS involvement in CLL is. Therefore, in this case report, we also conducted a comprehensive literature review of 50 case reports with CNS involvement in the last 10 years in which the clinical course was described. 


\section{Case Presentation}

A 33-year-old man with diplopia was referred to our hospital. He had a 9 month history of asymptomatic revised Rai low- and Binet A-stage CLL that had been diagnosed owing to an increase in lymphocyte count at a medical checkup, but he had not come to the hospital at his own discretion. Thereafter, he developed diplopia and was referred to neurosurgery by an ophthalmologist. Except for double vision and intracranial hypertension-related headaches, the neurological examination was unremarkable, and he had no other symptoms or lymph-node swelling. Magnetic resonance imaging (MRI) revealed a $5 \times 3.5 \mathrm{~cm}$ nonuniformly contrasted mass in the right temporal lobe that appeared hypointense on $T_{1}$-weighted and hyperintense on $T_{2}$-weighted images (Figure 1A). In this case, because there was a risk of cerebral hernia owing to a bulky CNS lesion, lumbar puncture could not be performed.

A diagnostic cranioscopic biopsy was performed, which revealed infiltration of small monoclonal lymphocytes with expression of CD5, CD20 (Figure 2), and CD79A, but without CD10, CD23, cyclin D1, or evidence of transformation. Similarly, his blood showed CLL-cell clonality, with expression of CD5, CD19, CD20 (dim), CD22, and cellsurface Ig, but no expression of CD10, CD23, or IgH-BCL1 on fluorescence in situ hybridization. Bone marrow (BM) specimens revealed $96.6 \%$ of lymphocytes had the same flow-cytometry appearance as peripheral blood (PB). BM lymphocytes had a normal karyotype without poor prognostic factors, deletion $17 \mathrm{p}$, deletion $11 \mathrm{q}$, or transformation (Figure 3), which was compatible with a diagnosis of CLL. These findings were indicative of leukemic involvement in the CNS, and the patient was eventually transferred to hematology. In this case, Richter's syndrome was initially suspected from the symptoms and course, but CNS-infiltrating cells were small lymphoid cells similar to those of PB and $\mathrm{BM}$, and transformation to a diffuse large-cell type was ruled out by brain biopsy. Therefore, we diagnosed CNS involvement in CLL.

Laboratory data (Table 1) were significant for a white blood-cell count of 464,200/ $\mu \mathrm{L}$ (98.5\% lymphocytes and $1.5 \%$ neutrophils). Hemoglobin level and platelet count were $11.7 \mathrm{~g} / \mathrm{dL}$ and $305,000 / \mu \mathrm{L}$, respectively. Lactate dehydrogenase was $262 \mathrm{IU} / \mathrm{L}$ (normal range 112-230 IU/L) and soluble IL2R 11,000 IU/L (normal range 124-466 IU/dL).
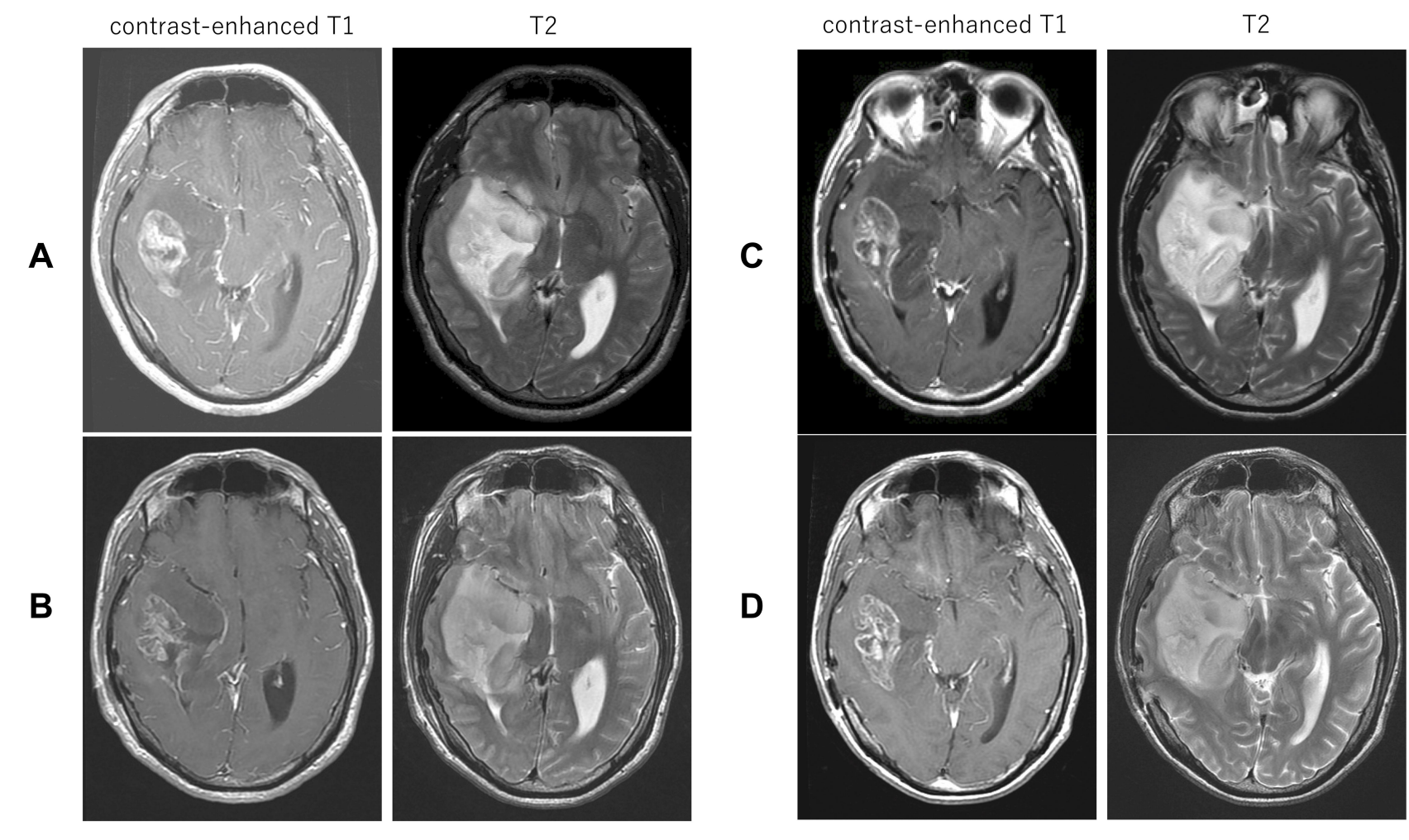

Figure I Magnetic resonance imaging (MRI) showing $5 \times 3.5 \mathrm{~cm}$ abnormal nonuniformly contrasted mass with hypointensity on $T_{1}$-weighted image (left) and with hyperintensity on $T_{2}$-weighted image (right) in the right temporal lobe. (A) MRI at first consultation; (B) MRI after MPV administration (at day I7 after admission); (C) MRI after FCR administration (at day 34 after admission); (D) MRI after lbr administration (at day 54 after admission). 

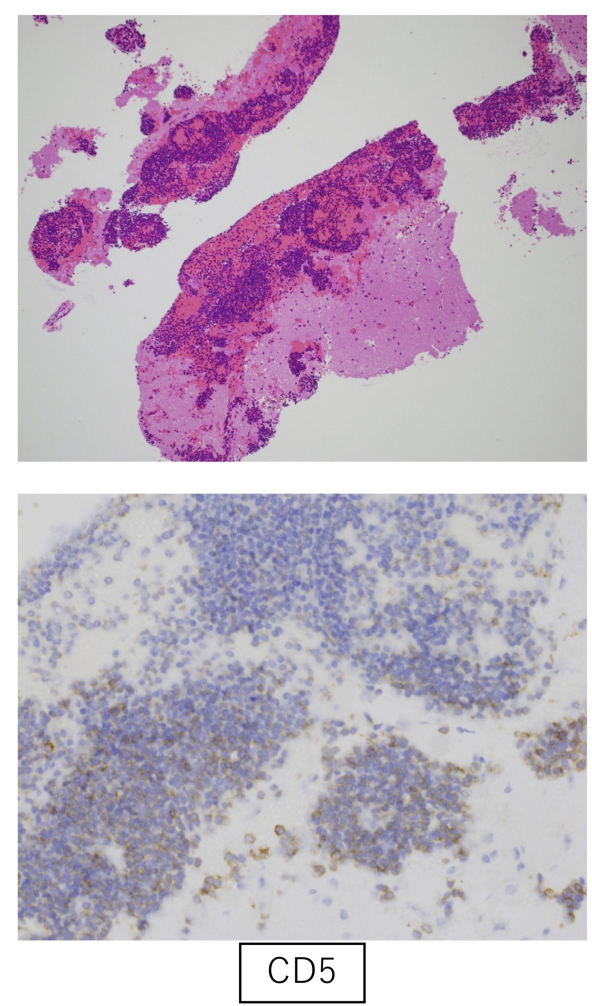
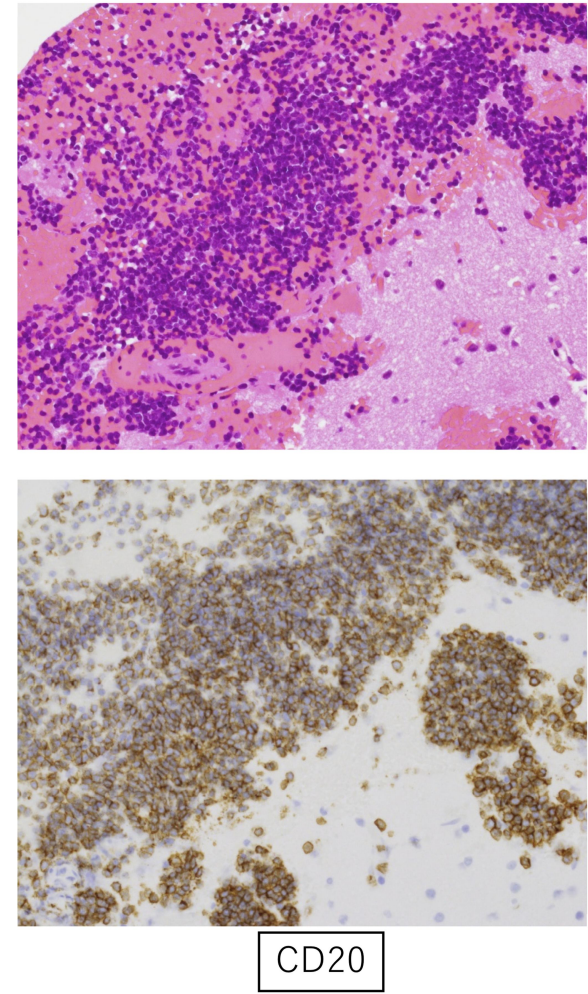

Figure 2 Brain specimens (cranioscopic biopsy) showing infiltration of small monoclonal lymphocytes with expression of CD5 and CD20 (upper left, H\&E $\times 40$; upper right, $\mathrm{H} \& \mathrm{E} \times 100$; lowerleft CD5 × 100; lower right, CD20 × 100).

$\beta_{2}$-microglobulin was $2.1 \mathrm{mg} / \mathrm{L}$. Evaluation with thoracoabdominal computed tomography (CT) revealed splenomegaly and mild systemic lymphadenopathy.

Treatment with $2 \mathrm{mg}$ betamethasone for 7 days transiently improved the diplopia and headaches, but tumor size evaluated by CT/MRI remained unchanged. No standard protocol exists for CLL with CNS involvement, because it is an extremely rare disease condition. Therefore, according to the treatment strategy of primary CNS lymphoma, MPV chemotherapy (methotrexate $3.5 \mathrm{mg} / \mathrm{m}^{2}$ on day 1 , vincristine $1.4 \mathrm{mg} / \mathrm{m}^{2}$ [max $2.8 \mathrm{mg}$ on day 1], and procarbazine $100 \mathrm{mg} / \mathrm{m}^{2}$ per day on days 1-7) was started. Ten days after treatment, intracranial hypertension-related symptoms, such as diplopia and headaches, recurred and performance status was decreased. MRI showed that the tumor size remained unchanged (Figure 1B) and PB-lymphocyte reduction was poor (Figure 4), indicating resistance to the MPV treatment. Therefore, rituximab (Rtx) $375 \mathrm{mg} / \mathrm{m}^{2}$ and subsequent whole-brain radiotherapy (30 Gy/15 fr) plus simultaneous in-field boost (10 Gy/5 fr) were administered.

After Rtx administration, the diplopia and headaches improved and lymphocyte reduction was observed.
Therefore, treatment with one cycle of FCR chemotherapy (fludarabine $25 \mathrm{mg} / \mathrm{m}^{2}$ per day and cyclophosphamide $250 \mathrm{mg} / \mathrm{m}^{2}$ per day for the first 3 days, with addition of Rtx $375 \mathrm{mg} / \mathrm{m}^{2}$ ) was started. Although the PB lymphocytes decreased steadily (Figure 4) without recurrence of intracranial hypertension-related symptoms, no reductive effect on the intracranial tumor was observed on contrast-enhanced MRI (Figure 1C). Because the effects of ibrutinib (Ibr) on the CNS have been reported in CLL and mantle-cell lymphoma, ${ }^{6}$ we next selected Ibr $420 \mathrm{mg} /$ day for treatment. However, 2 weeks later, contrast-enhanced MRI revealed no reductive effect, and diplopia and headaches had recurred (Figure 1D). Finally, the patient refused subsequent treatment and was self-discharged from the hospital. He died at home 9 weeks after the onset of initial symptoms (48 weeks after the diagnosis of CLL).

\section{Discussion}

Diagnostic cranioscopic biopsy was performed in our case, but many cases were diagnosed by cerebrospinal fluid (CSF) analysis in a retrospective cohort of $30 \mathrm{CLL}$ patients with CNS involvement. ${ }^{7}$ In that cohort, biopsies 


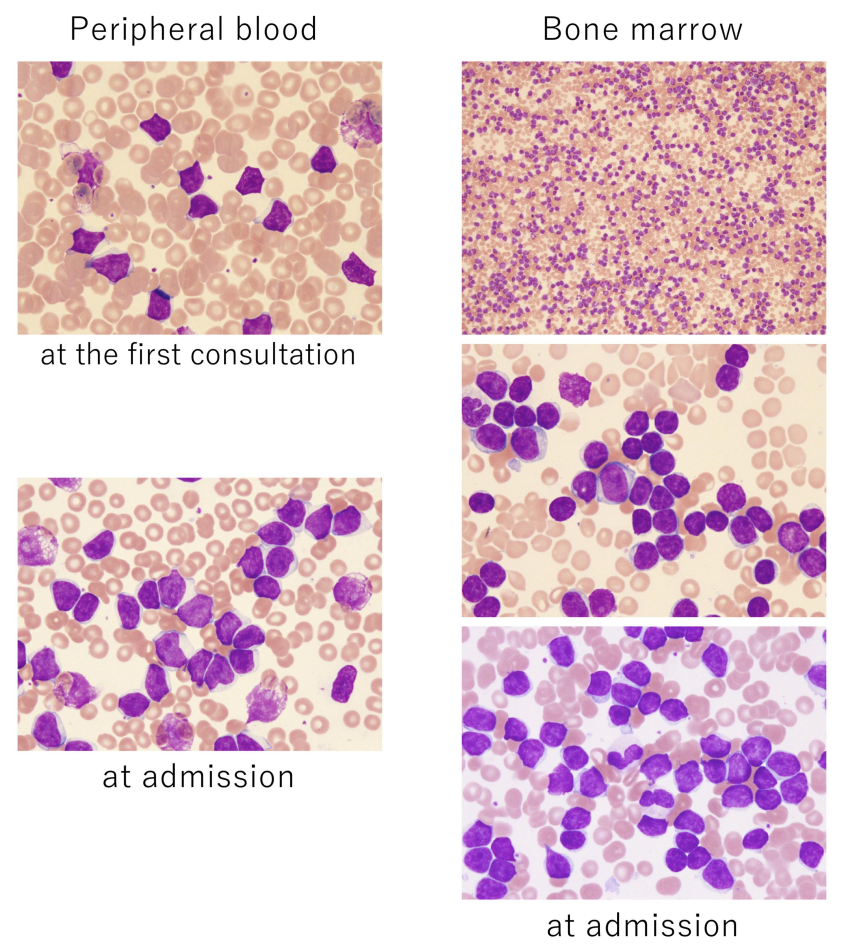

Figure $3 \mathrm{CLL}$ cells from peripheral blood at first consultation and admission and BM at admission showed mature small monoclonal lymphocytes with narrow cytoplasm, concentrated nuclei, and partially aggregated chromatin without transformation to a large cell type (H\&E $\times 40$ and $\times 200)$.

were performed in only five cases, of which only one was diagnosed by brain biopsy. ${ }^{7}$ Our review of the literature revealed diagnostic biopsies had been performed in 12 of 50 cases (not including surgical resection). Ten of the 50 cases were diagnosed as Richter's syndrome, and $11.3 \%$ of Richter's transformation with intracranial involvement was found in an old literature review of CLL (before 2011). ${ }^{8}$ By contrast, there were no cases of Richter's syndrome in the 30 cases of the retrospective cohort. ${ }^{7}$ It has been reported that Richter's transformation occurs in approximately $5 \%-10 \%$ of the CLL population; ${ }^{9}$ therefore, it is still difficult to conclude whether there is an intimate correlation between CNS involvement and Richter's transformation.

The 50 reported cases of CNS involvement in CLL had diverse and uncharacteristic symptoms, such as headaches, convulsions, diplopia, ataxia, facial paralysis, and cognitive dysfunction (Table 2). It is difficult to identify the risk factors for CNS involvement in CLL. ${ }^{10,11}$ Our literature review confirmed this, because we could not find a common feature in cases of CNS involvement. There are cases in which CNS involvement develops when the stage is not necessarily progressive (on Rai or
Table I Hematologic Assessment of Patient

\begin{tabular}{|c|c|c|c|}
\hline White blood Cells/ $\mu \mathrm{L}$ & $464,200 / \mu \mathrm{L}$ & $\mathbf{N a}$ & $141 \mathrm{mEq} / \mathrm{L}$ \\
\hline Neutrophils & $1.5 \%$ & K & $4.2 \mathrm{mEq} / \mathrm{L}$ \\
\hline Basophils & 0 & $\mathrm{Cl}$ & $103 \mathrm{mEq} / \mathrm{L}$ \\
\hline Eosinophils & 0 & BUN & $12 \mathrm{mg} / \mathrm{dL}$ \\
\hline Lymphocytes & $98.5 \%$ & $\mathrm{Cr}$ & $0.83 \mathrm{mg} / \mathrm{dL}$ \\
\hline Monocytes & 0 & TP & $6.5 \mathrm{~g} / \mathrm{dL}$ \\
\hline Others & 0 & Alb & $4.1 \mathrm{~g} / \mathrm{dL}$ \\
\hline Plt & $30.5 \times 10^{4} / \mu \mathrm{L}$ & AST & $26 U / L$ \\
\hline RBC & $449 \times 10^{4} / \mu \mathrm{L}$ & ALT & $37 \mathrm{U} / \mathrm{L}$ \\
\hline $\mathrm{Hb}$ & $11.7 \mathrm{~g} / \mathrm{dL}$ & T-Bil & $0.4 \mathrm{mg} / \mathrm{dL}$ \\
\hline $\mathrm{Ht}$ & $42.1 \%$ & D-Bil & $0 \mathrm{mg} / \mathrm{dL}$ \\
\hline MCV & $93.8 \mathrm{fL}$ & ALP & $544 \mathrm{U} / \mathrm{L}$ \\
\hline $\mathrm{MCH}$ & $26.1 \mathrm{pg}$ & $\gamma$ GTP & $87 \mathrm{U} / \mathrm{L}$ \\
\hline $\mathrm{MCHC}$ & $27.8 \mathrm{~g} / \mathrm{dL}$ & LDH & $262 \mathrm{U} / \mathrm{L}$ \\
\hline APTT & 25.7 seconds & CRP & $0.072 \mathrm{mg} / \mathrm{dL}$ \\
\hline PT & $107.4 \%$ & & \\
\hline FBG & $241 \mathrm{mg} / \mathrm{dL}$ & $\lg G$ & $955 \mathrm{mg} / \mathrm{dL}$ \\
\hline AT-III & $109 \%$ & $\lg A$ & $83 \mathrm{mg} / \mathrm{dL}$ \\
\hline $\mathrm{HBs}-\mathrm{Ag}$ & - & $\lg M$ & $29 \mathrm{mg} / \mathrm{dL}$ \\
\hline HCV-Ab & - & sIL2R & 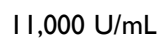 \\
\hline HTLV-I & - & BMG & $2.1 \mathrm{mg} / \mathrm{L}$ \\
\hline HIV & - & ANA & - \\
\hline
\end{tabular}

Binet staging) or without high-risk chromosomal abnormality, such as del17p or del11q. This suggests clinical and pathophysiological heterogeneity of CNS involvement in CLL. ${ }^{7}$

A report summarizing the literature published before 2011 of CNS involvement in $\mathrm{CLL}^{8}$ showed average age 63.4 years, average latency between CLL diagnosis and first signs of CNS involvement 2.6 years, average overall survival (OS) from CLL diagnosis 3.8 years, and average OS from time of CNS onset 12 months. Our review of the 50 case reports revealed average age 62.2 years (in 49 cases) and average latency 4.9 years (in 32 cases. OS data could not be extracted. Our case showed a younger and more aggressive disease course of age 33 years, latency 9 months, OS 48 weeks, and OS from time of CNS onset 9 weeks. Our case was resistant to high-dose Mtx and whole-brain radiotherapy as standard treatments for primary CNS lymphoma. As the standard treatment for non-high risk CLL, FCR was effective in reducing the number of $\mathrm{PB}$ lymphocytes and improved intracranial hypertension-related symptoms; however, it had less effect on tumor shrinkage, indicating it was ineffective for the CNS lesion. Although the number of reports of CNS involvement in CLL is low, there were reports of successful treatment with FCR in some cases in our 

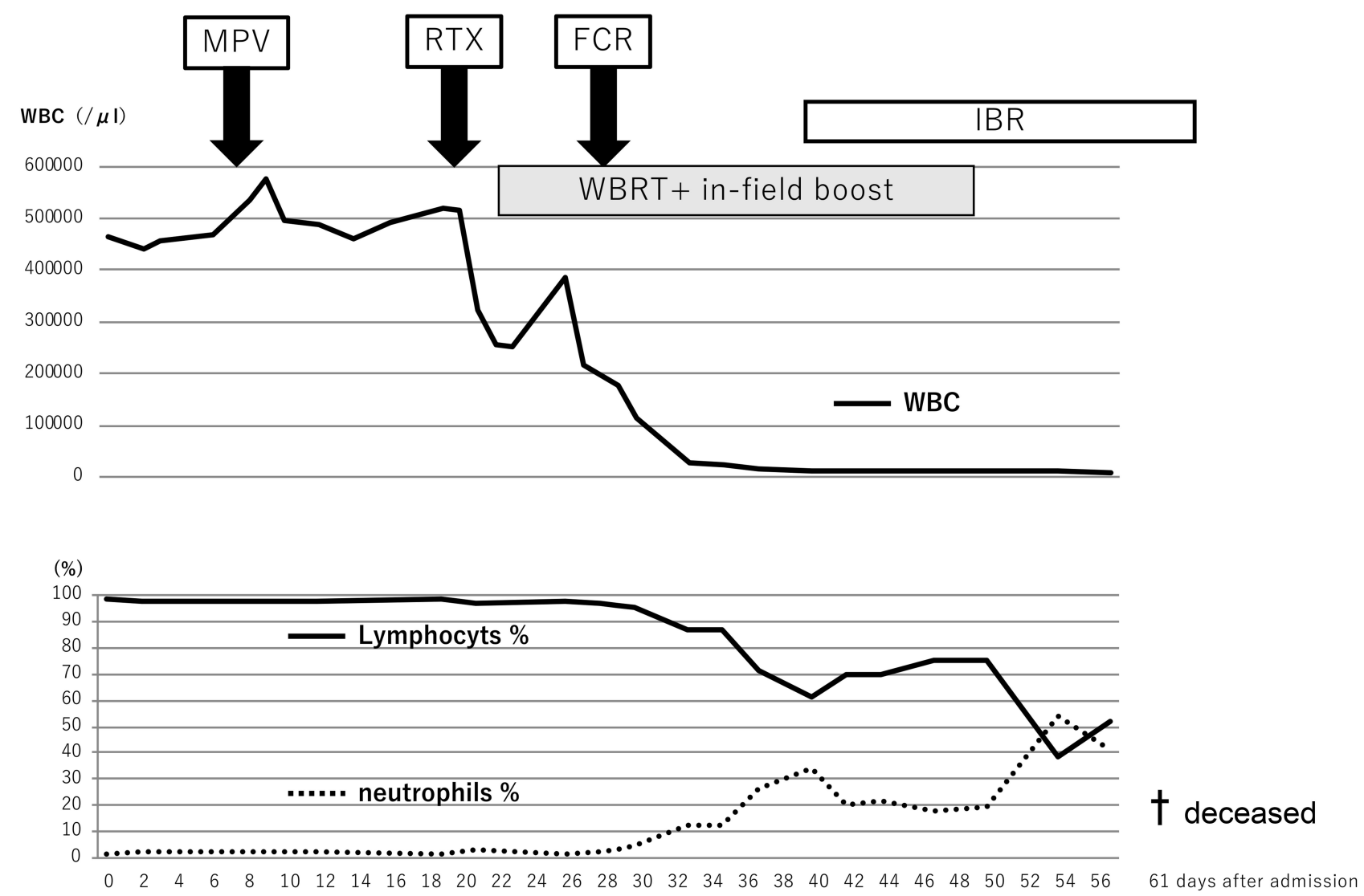

Figure 4 Clinical course of WBC and lymphocyte counts after treatment.

Abbreviations: MPV, methotrexate $3.5 \mathrm{mg} / \mathrm{m}^{2}$ (day I), vincristine $1.4 \mathrm{mg} / \mathrm{m}^{2}$ (max $2.8 \mathrm{mg}$ ) (day I), and procarbazine $100 \mathrm{mg} / \mathrm{m}^{2}$ per day (days I-7); Rtx, rituximab (375 mg/ $\mathrm{m}^{2}$; FCR, fludarabine $\left(25 \mathrm{mg} / \mathrm{m}^{2}\right.$ per day), cyclophosphamide $\left(250 \mathrm{mg} / \mathrm{m}^{2}\right.$ per day) for the first 3 days, with addition of Rtx $375 \mathrm{mg} / \mathrm{m}^{2}$; WBRT, whole-brain radiotherapy $(30$ $\mathrm{Gy} / \mathrm{I} 5 \mathrm{fr}$ ) plus simultaneous in-field boost (10 Gy/5 fr).

literature review (Table 2). However, in general, prognosis was poor, owing to resistance to various treatments, such as high-dose Mtx, intrathecal injection, whole-brain radiotherapy, and FCR. A similar result was obtained in our case

Ibr has been reported to be effective in CNS lesions of mantle-cell lymphoma ${ }^{6}$ and Waldenström macroglobulinemia. ${ }^{12}$ Effects of Ibr appear 1-2 weeks after administration. ${ }^{13}$ Nine successful cases of Ibr treatment for CNS involvement in CLL were found in 50 cases (complete response in eight cases, partial response in one) (Table 2); therefore, Ibr may be a promising drug for CNS involvement. However, this was not found in our case. It is possible that the effective concentration of Ibr in the CNS lesion had not reached sufficient levels in our case. Concentration in CSF was reported to be $2 \log$ lower than in the plasma of 18 patients with primary CNS lymphoma treated with Ibr. $^{14}$ It has been reported that an increased dose of Ibr escalates CSF concentration without adverse events, ${ }^{15}$ and that increasing the dose of Ibr is effective for CNS lesions in CLL. ${ }^{16}$ It will be necessary in the future to verify the optimal dose of Ibr for CNS lesions. Bulky disease in CNS lesion might also cause treatment failure. Although ofatumumab and alemtuzumab are alternative treatment options, we did not select them, because they did not show superiority to Ibr in the data or in drug penetration of the CNS. In addition, there have been two reports showing the effectiveness of venetoclax against CNS lesions (Table 2). ${ }^{17,18}$ One of those was a case where venetoclax was effective after Ibr resistance, and thus it may be beneficial to test venetoclax against CNS involvement in CLL.

\section{Conclusion}

Patients with CNS lesions in lymphoid tumors have a poor prognosis, but the possibility of concomitant use of Mtx and Ibr or venetoclax can be envisioned. Accumulation of 


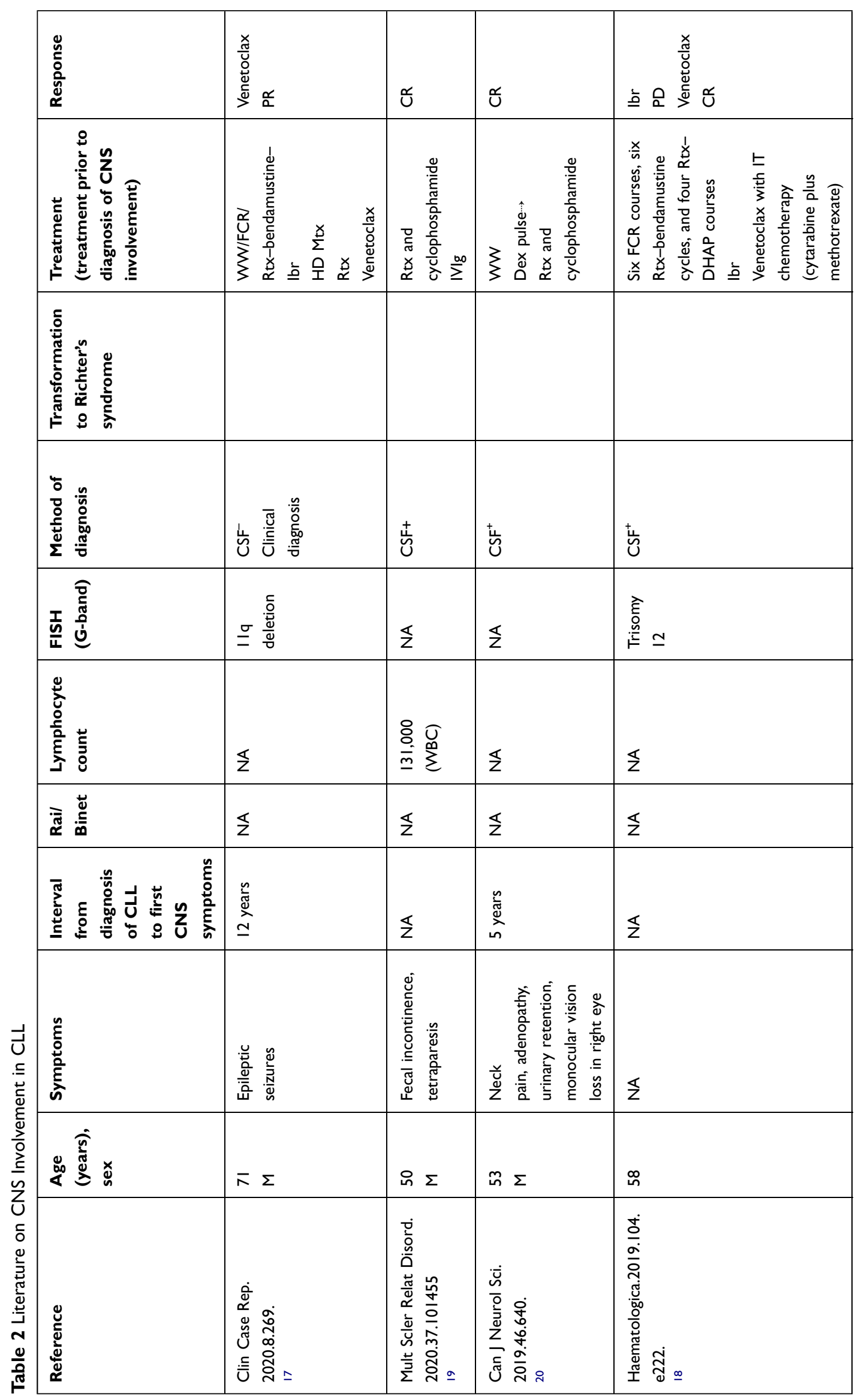




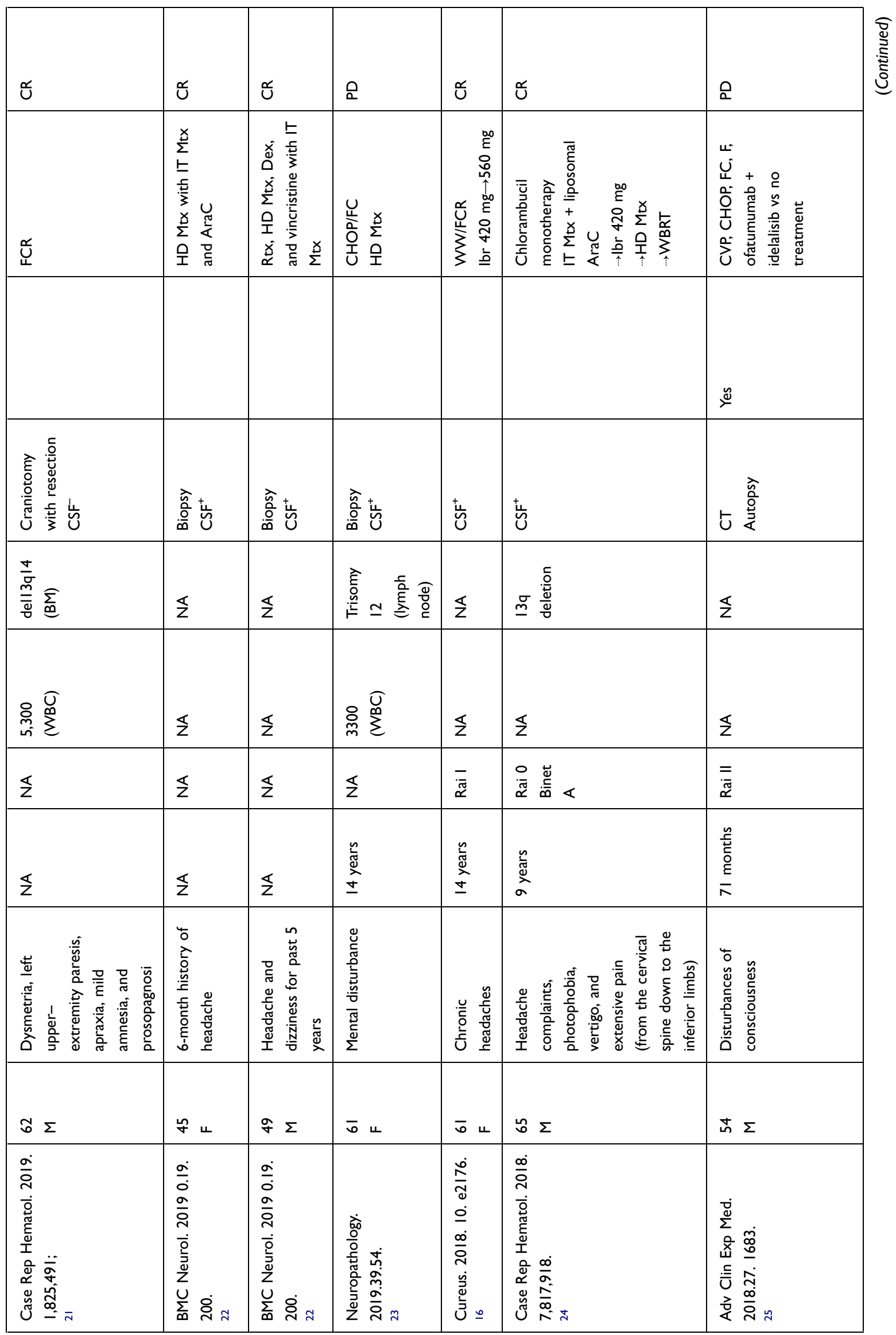




\begin{tabular}{|c|c|c|c|c|c|c|}
\hline 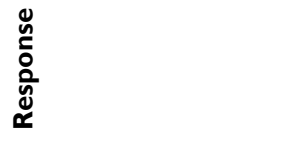 & $\dddot{\alpha}$ & $\stackrel{\alpha}{a}$ & 은 & 은 & 은 & 은 \\
\hline 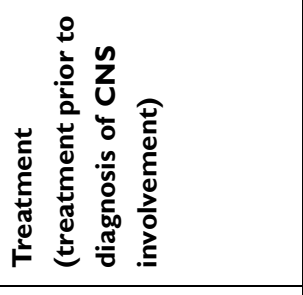 & 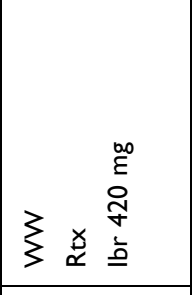 & 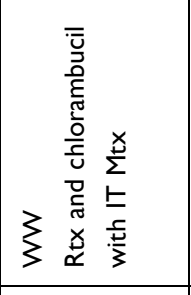 & 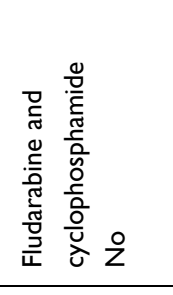 & 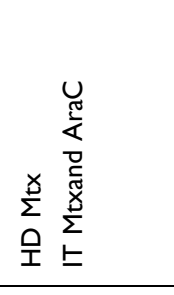 & 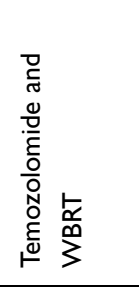 & 主紊 \\
\hline 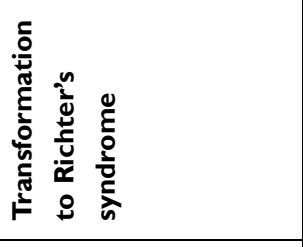 & & & & $\stackrel{\mathscr{g}}{=}$ & $\stackrel{\mathscr{g}}{\bullet}$ & \\
\hline 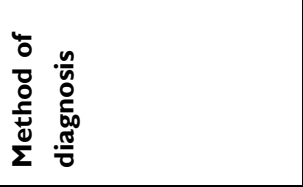 & 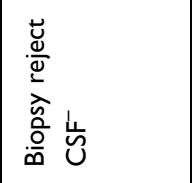 & 㺃 & 萢 & 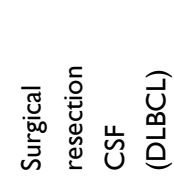 & 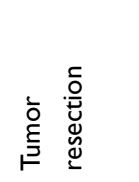 & 萢 \\
\hline 窟 & \begin{tabular}{|l}
$\overline{\bar{g}}$ \\
$\overline{\tilde{g}}$ \\
$\bar{z}$
\end{tabular} & $\frac{\pi}{z}$ & $\frac{\pi}{z}$ & 确 & $\underline{z}$ & $\frac{\stackrel{\rho}{\bar{v}}}{\bar{v}}$ \\
\hline 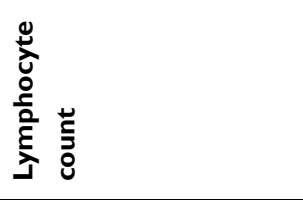 & $\begin{array}{l}\tilde{\tilde{S}} \\
\dot{\tilde{f}} \\
\dot{q}\end{array}$ & 惫 & 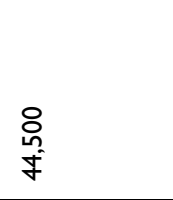 & 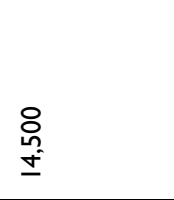 & 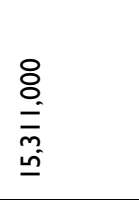 & $\begin{array}{l}80 \\
0 \\
0 \\
0.0 \\
\infty \\
\infty\end{array}$ \\
\hline 离产 & 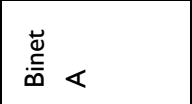 & 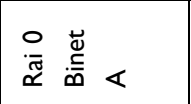 & 竞 & $\mathbb{z}$ & $\stackrel{\circ}{\stackrel{\circ}{\bar{x}}}$ & 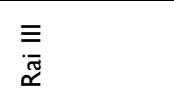 \\
\hline 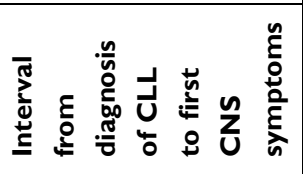 & 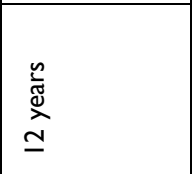 & 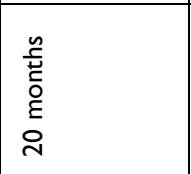 & 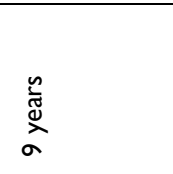 & $\mathbb{Z}$ & $\underline{z}$ & 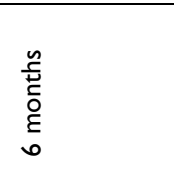 \\
\hline 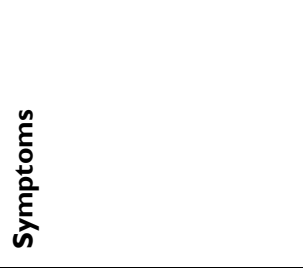 & 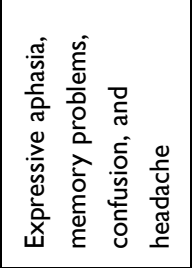 & 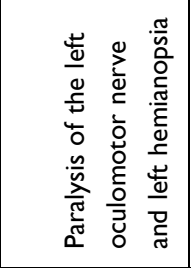 & 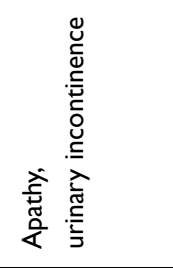 & 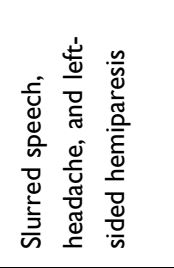 & 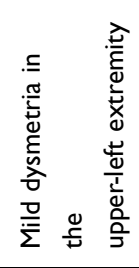 & 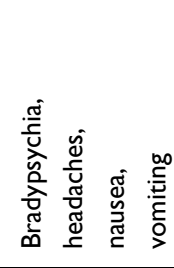 \\
\hline 离 & $\Sigma_{4}$ & $\bar{\infty} \Sigma$ & $R \Sigma$ & tิ & Б & in $\Sigma$ \\
\hline 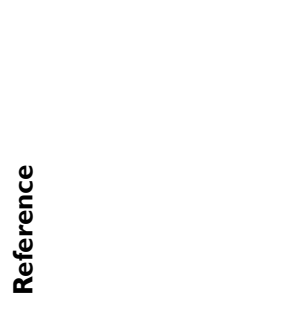 & 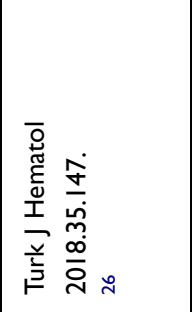 & 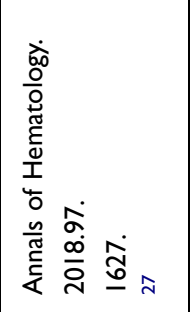 & 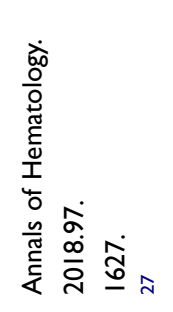 & 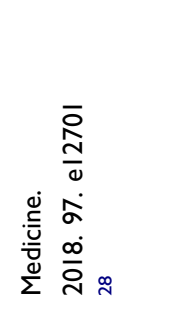 & 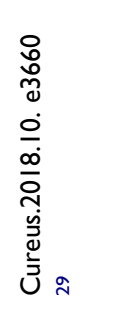 & 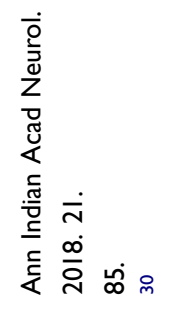 \\
\hline
\end{tabular}




\begin{tabular}{|c|c|c|c|c|c|c|c|}
\hline ồ & Q & $\mathrm{Q}$ & Ů & $\begin{array}{l}\frac{0}{a} \\
\frac{1}{\alpha} \\
\frac{\alpha}{\alpha}\end{array}$ & Ư & Ư & Ư \\
\hline U Ư⿱艹亡 & $\varliminf_{3}^{\frac{0}{1}}$ & 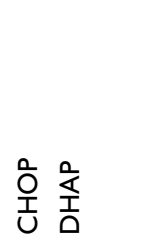 & 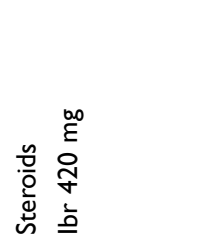 & 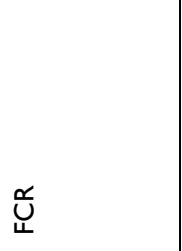 & $\begin{array}{l}\frac{x}{x} \\
上 \\
\frac{5}{5} \\
\frac{5}{3} \\
\vdots \\
\underline{\underline{t}}\end{array}$ & 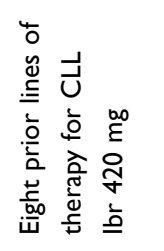 & 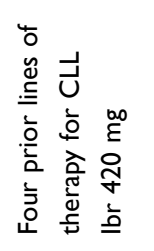 \\
\hline 苞 & 岕 & 苍 & 苅 & 苍 & 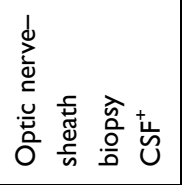 & 岕 & 芯 \\
\hline $\begin{array}{l}\overline{\widetilde{g}} \\
\text { है } \\
\text { z }\end{array}$ & 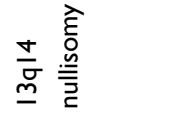 & $\S$ & $\S$ & $\begin{array}{l}\overline{\widetilde{g}} \\
\text { है } \\
\text { z }\end{array}$ & $\S$ & 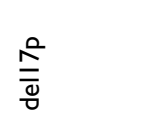 & $\frac{\stackrel{2}{\bar{v}}}{\frac{\bar{v}}{0}}$ \\
\hline 兽 & 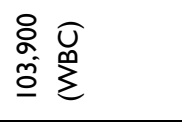 & $\begin{array}{l}8 \\
0 \\
0 \\
0 \\
\infty \\
\infty \\
\infty\end{array}$ & $\begin{array}{l}\text { ठ } \\
\text { ホं }\end{array}$ & $\begin{array}{l}\stackrel{8}{+} \\
\stackrel{+}{-}\end{array}$ & 啇 & $\S$ & 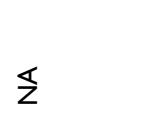 \\
\hline $\begin{array}{l}\geq \overline{\widetilde{\sigma}} \\
\stackrel{\alpha}{\alpha}\end{array}$ & 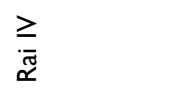 & $=$ & $\underset{\substack{\overline{\widetilde{\sigma}} \\
\text { 三 }}}{\equiv}$ & $\stackrel{\circ}{\circ}$ & $\S$ & 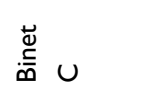 & 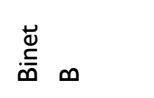 \\
\hline 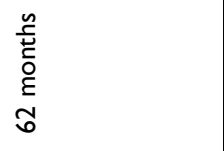 & $\begin{array}{l}\frac{n}{c} \\
\dot{\vec{L}} \\
\stackrel{0}{\xi} \\
\sigma\end{array}$ & 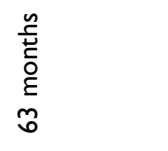 & $\S$ & $\S$ & 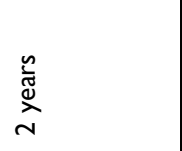 & $\Sigma$ & $\S$ \\
\hline 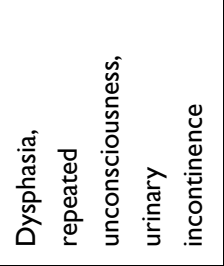 & 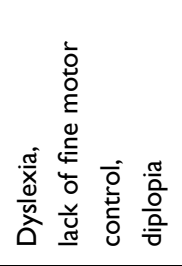 & 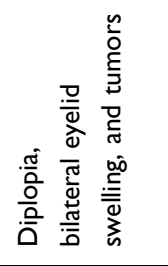 & 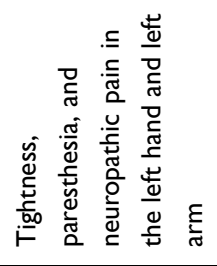 & 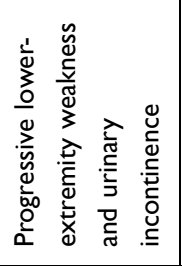 & 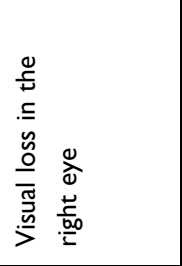 & 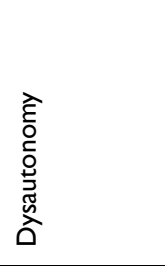 & 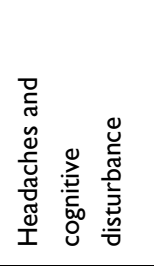 \\
\hline $\mathcal{F} \Sigma$ & $\approx \Sigma$ & $q \delta$ & $\stackrel{\circ \Sigma}{ }$ & 8. & $\mathscr{f} \Sigma$ & $\stackrel{\infty}{n} \Sigma$ & $\stackrel{n}{\wedge} \Sigma$ \\
\hline 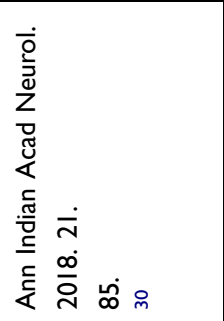 & 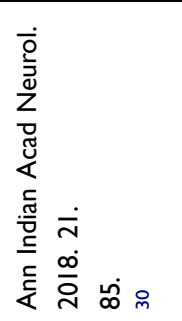 & 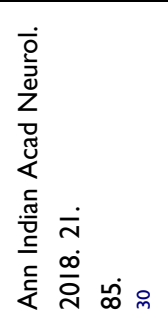 & 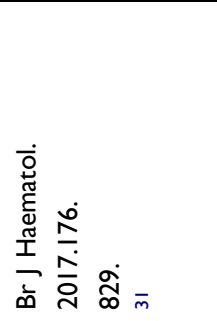 & 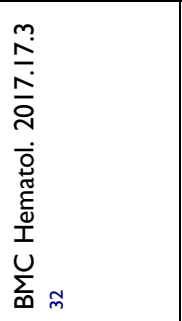 & 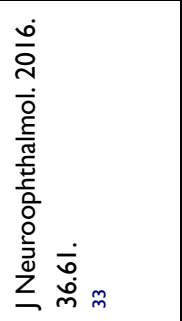 & 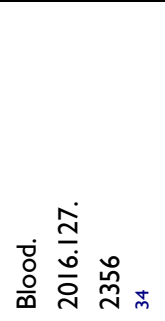 & 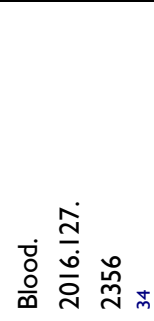 \\
\hline
\end{tabular}




\begin{tabular}{|c|c|c|c|c|c|c|}
\hline 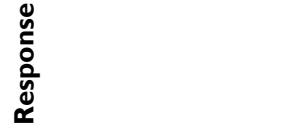 & $\widetilde{U}$ & $\tilde{U}$ & Ů & Ư & 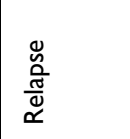 & ư \\
\hline 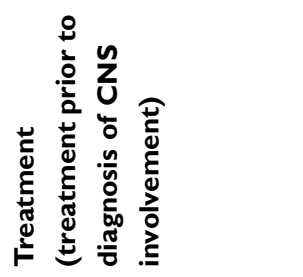 & 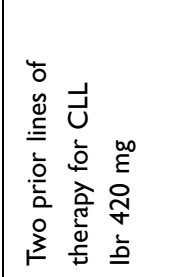 & 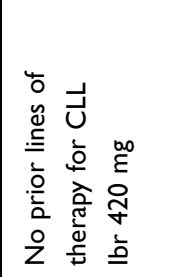 & 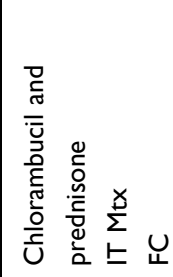 & 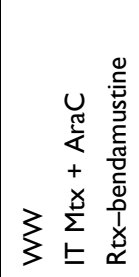 & 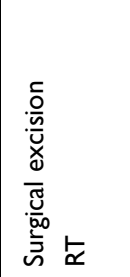 & |⿹ \\
\hline 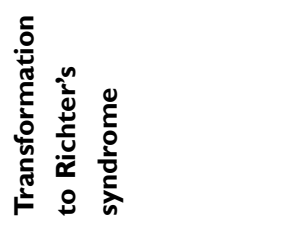 & & & & & & \\
\hline 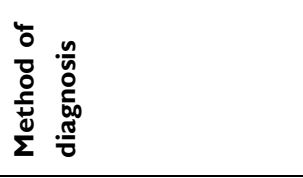 & 苞 & 荡 & 荡 & 苟 & 商 & 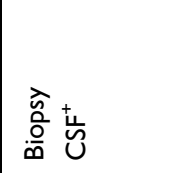 \\
\hline 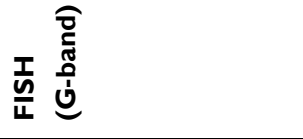 & $\mathbb{z}$ & $\frac{\varrho}{\overline{\bar{g}}}$ & $\frac{\pi}{z}$ & \begin{tabular}{|l}
$\overline{\bar{g}}$ \\
$\overline{\underline{\underline{g}}}$ \\
z
\end{tabular} & $\frac{\pi}{z}$ & 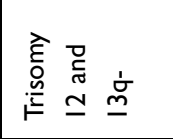 \\
\hline 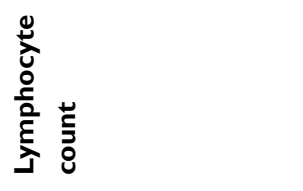 & $\mathbb{Z}$ & $\frac{\mathbb{s}}{z}$ & 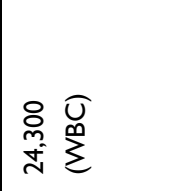 & $\frac{8}{\dot{i}}$ & $\frac{\mathbb{s}}{z}$ & $\mid \begin{array}{l}0 \\
\substack{0 \\
\infty \\
\infty \\
o}\end{array}$ \\
\hline 离 & 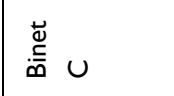 & 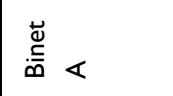 & $\overline{\overline{\bar{\alpha}}}$ & ه & $\underline{s}$ & $\overline{\overline{\tilde{\Phi}}}$ \\
\hline 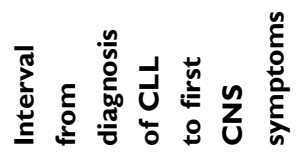 & $\mathbb{z}$ & $\underline{z}$ & 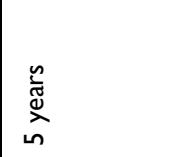 & 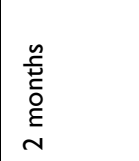 & $\underline{z}$ & 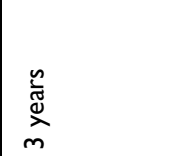 \\
\hline 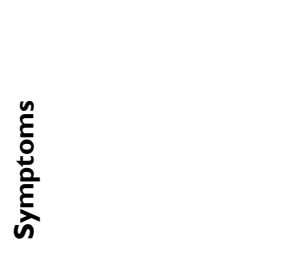 & 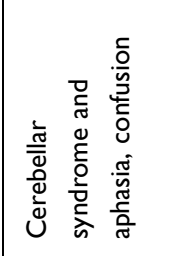 & 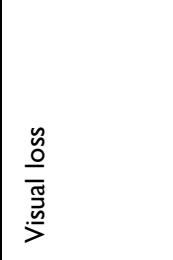 & 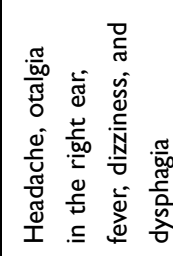 & 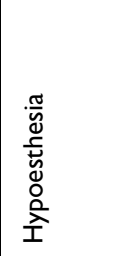 & 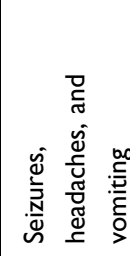 & 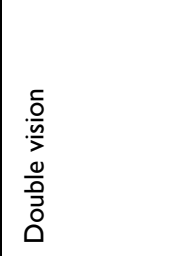 \\
\hline 品哭 & $0 \Sigma$ & $\infty_{\circ}$ & 는 & $\Phi \Sigma$ & ๖ ะ & まะ \\
\hline 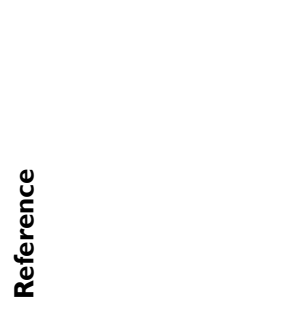 & 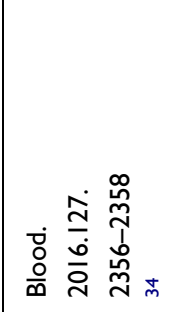 & 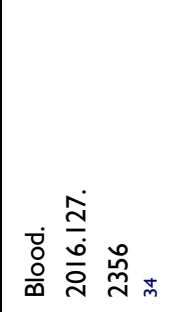 & 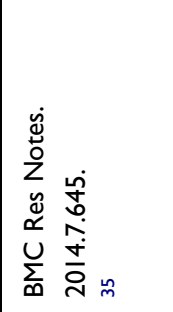 & 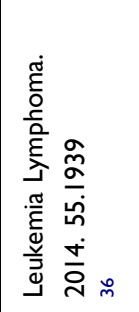 & 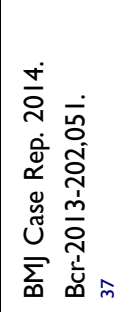 & 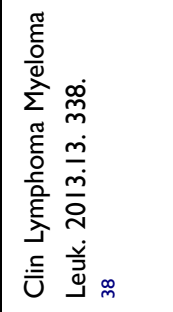 \\
\hline
\end{tabular}




\begin{tabular}{|c|c|c|c|c|}
\hline O & & 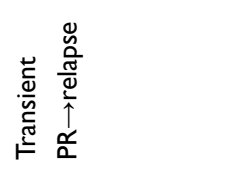 & Ů & 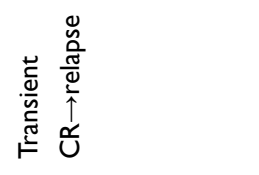 \\
\hline 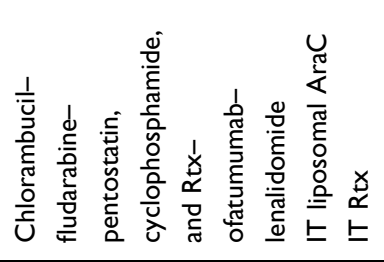 & 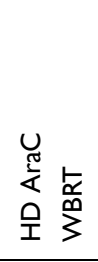 & 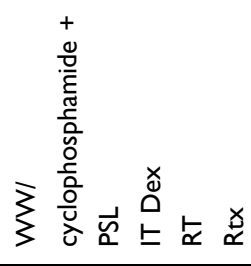 & 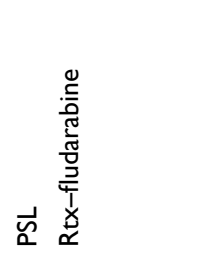 & 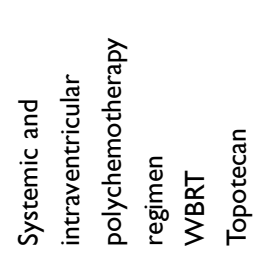 \\
\hline$\stackrel{\Xi}{\nu}$ & & $\stackrel{\Xi}{\check{\Perp}}$ & & $\stackrel{\varpi}{\nu}$ \\
\hline 苞 & & 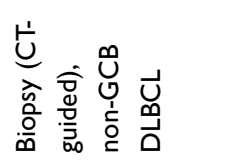 & 苐 & 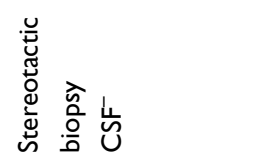 \\
\hline$\frac{\frac{0}{0}}{\frac{0}{0}}$ & & $\mathbb{z}$ & 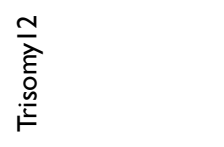 & $\mathbb{z}$ \\
\hline 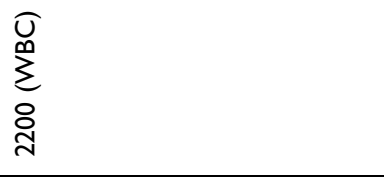 & & $\begin{array}{l}\text { ¿ } \\
\text { ন }\end{array}$ & 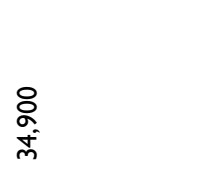 & 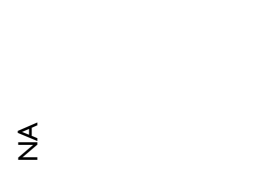 \\
\hline$\overline{\overline{\widetilde{g}}}$ & & $\begin{array}{l}\circ \\
\stackrel{\bar{\sigma}}{\bar{\alpha}}\end{array}$ & $\begin{array}{l}\circ \\
\stackrel{\overline{\widetilde{x}}}{\propto}\end{array}$ & 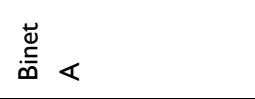 \\
\hline 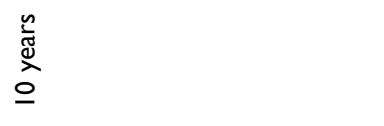 & & 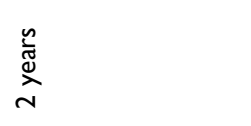 & 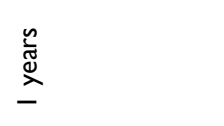 & 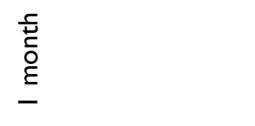 \\
\hline 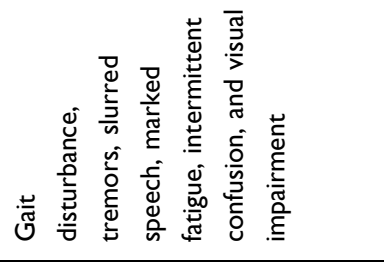 & & 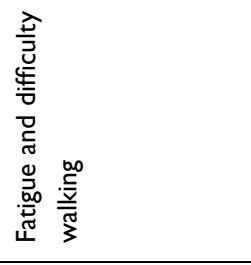 & 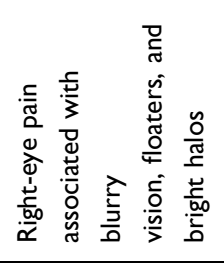 & 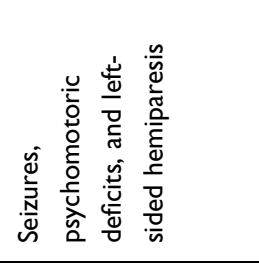 \\
\hline$\hat{\sigma} \Sigma$ & & $\stackrel{\circ}{\circ}$ & $\stackrel{n}{\wedge} \Sigma$ & i̊ $\Sigma$ \\
\hline 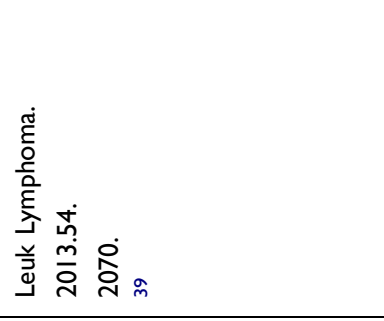 & & 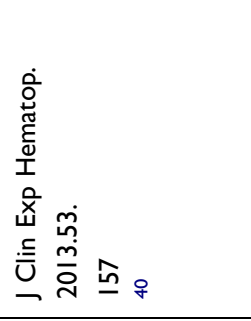 & 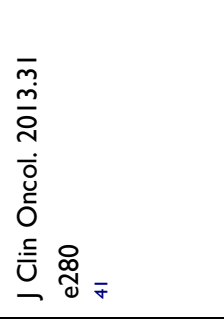 & 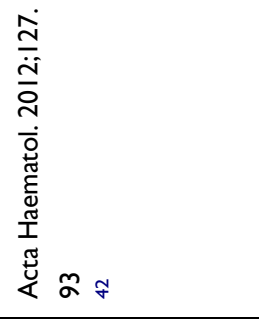 \\
\hline
\end{tabular}




\begin{tabular}{|c|c|c|c|c|c|}
\hline 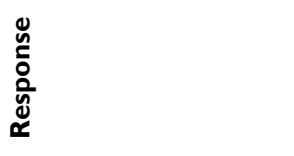 & $\stackrel{\propto x}{ }$ & 음 & Ư & $\stackrel{\alpha}{\alpha}$ & 음 \\
\hline 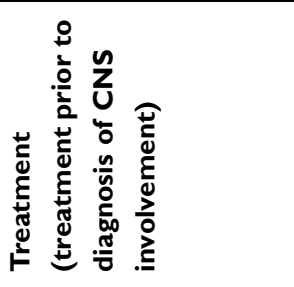 & 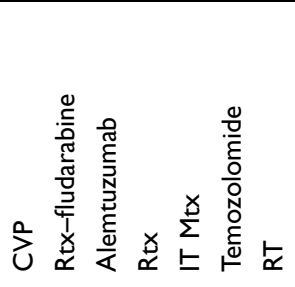 & 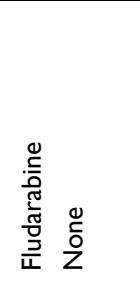 & 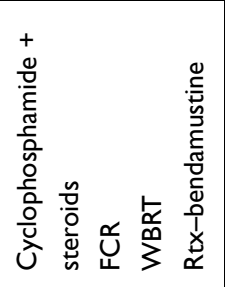 & 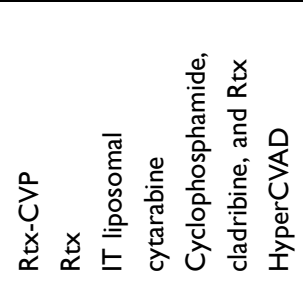 & 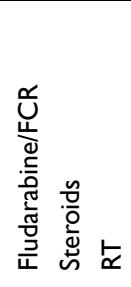 \\
\hline 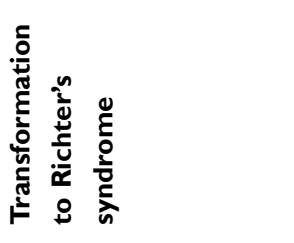 & & & & & \\
\hline 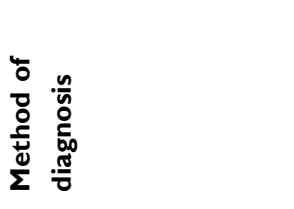 & 荡 & 葛 & $\begin{array}{l}\frac{\hat{े}}{0} \\
\frac{o}{0}\end{array}$ & 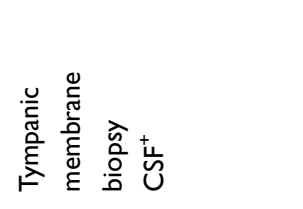 & 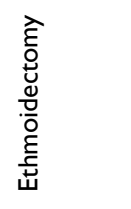 \\
\hline 至 & $\overleftarrow{z}$ & $\mathbb{z}$ & 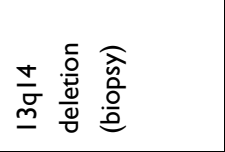 & 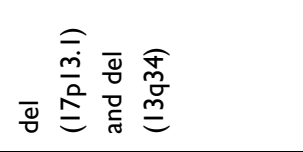 & $\Sigma$ \\
\hline 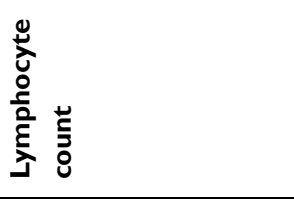 & 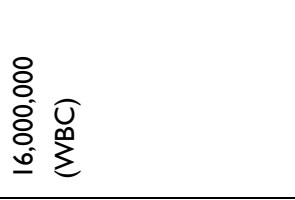 & 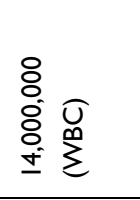 & 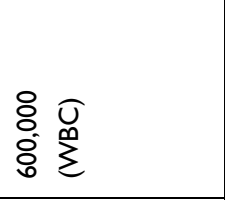 & 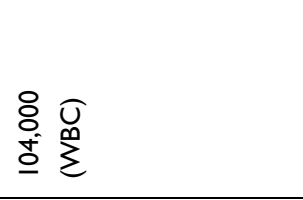 & $\underline{z}$ \\
\hline 离 & $\overline{\overline{\widetilde{\widetilde{\alpha}}}}$ & $\underset{\substack{\tilde{\tilde{\sigma}} \\
\propto}}{\geq}$ & 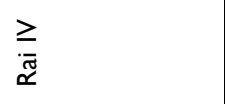 & $\underline{z}$ & 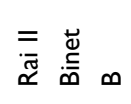 \\
\hline 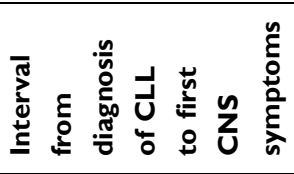 & 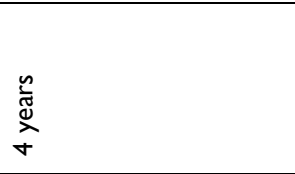 & 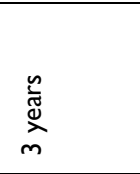 & $\begin{array}{l}\text { 䇏 } \\
\underline{\underline{\partial}} \\
\underline{E} \\
\end{array}$ & $\Sigma$ & $\mathbb{z}$ \\
\hline 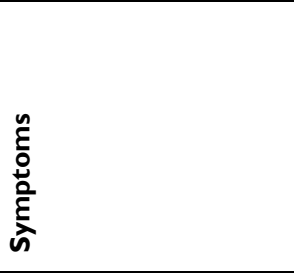 & 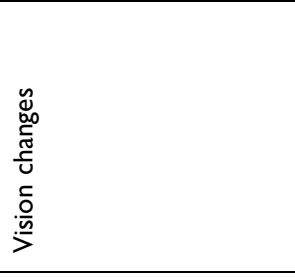 & 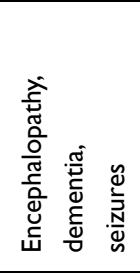 & 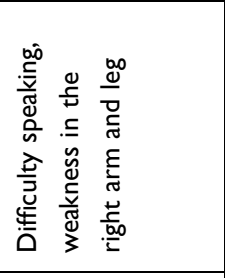 & 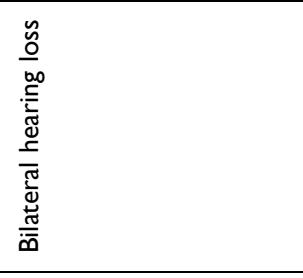 & 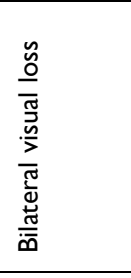 \\
\hline 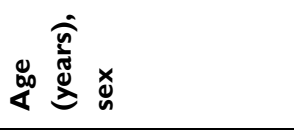 & ñ & $\approx \Sigma \Sigma$ & ถุ & ○ ะ & n \\
\hline 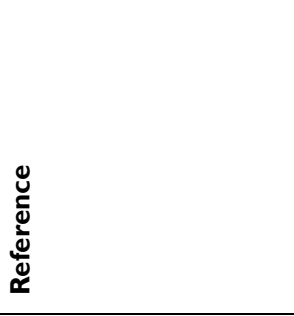 & 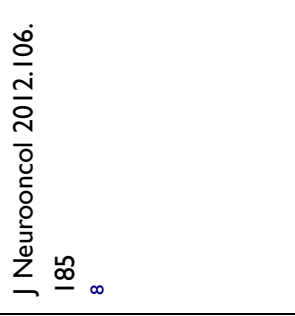 & 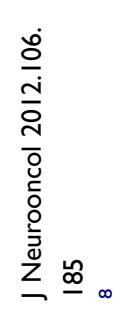 & 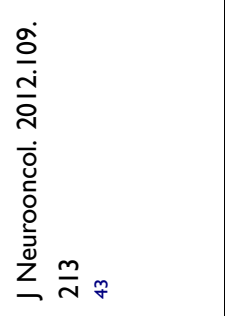 & 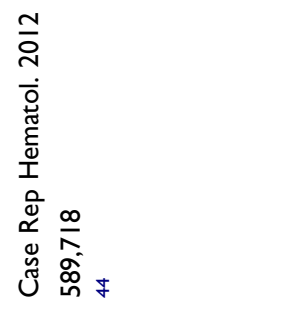 & 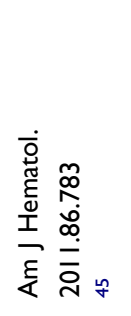 \\
\hline
\end{tabular}




\begin{tabular}{|c|c|c|c|c|c|c|c|}
\hline $\mathrm{Q}$ & $\begin{array}{l}\stackrel{n}{+} \\
\stackrel{5}{0} \\
\stackrel{0}{E} \\
0\end{array}$ & Ư & Ư & 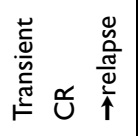 & 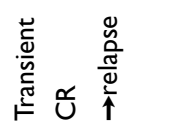 & Ư & Ũ \\
\hline 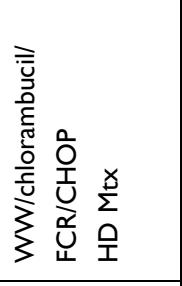 & $\frac{x}{\Sigma}$ 른 도 & 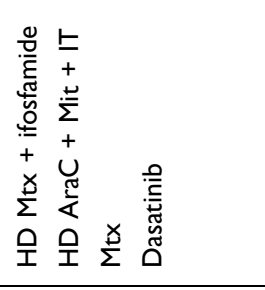 & 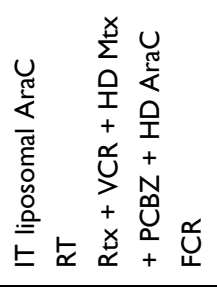 & 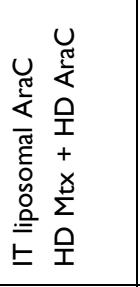 & 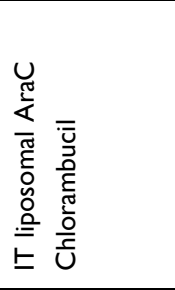 & 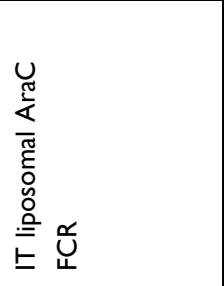 & 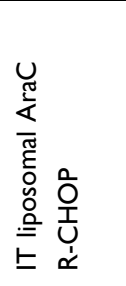 \\
\hline$\stackrel{\check{\nu}}{\check{\nu}}$ & & & & $\stackrel{y}{\check{\nu}}$ & & & \\
\hline 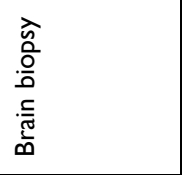 & 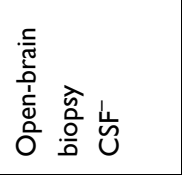 & 苍 & 苞 & 苞 & 药 & 峛 & 怱 \\
\hline $\begin{array}{l}\overline{\widetilde{\sigma}} \\
\text { हे } \\
\text { z }\end{array}$ & 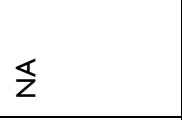 & $\S$ & $\S$ & 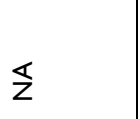 & $\S$ & $\S$ & $\S$ \\
\hline$\S$ & 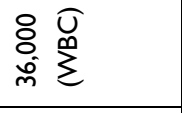 & 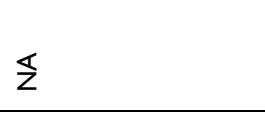 & $\S$ & $\nwarrow$ & $\nwarrow$ & $\S$ & $\S$ \\
\hline 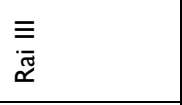 & $\begin{array}{l}\bar{c} \\
\check{\check{x}} \\
\end{array}$ & 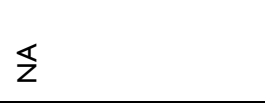 & 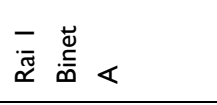 & 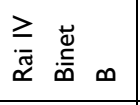 & 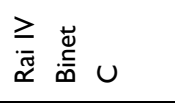 & 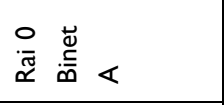 & 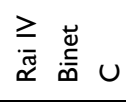 \\
\hline$\S$ & 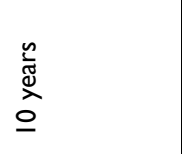 & $\S$ & 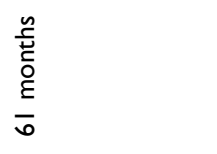 & 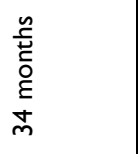 & 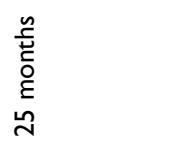 & 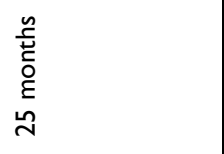 & 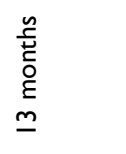 \\
\hline 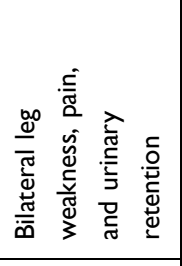 & 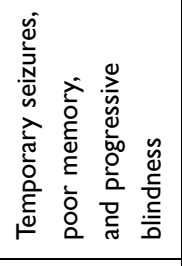 & 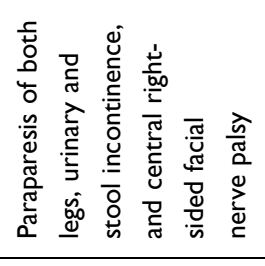 & 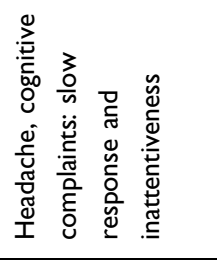 & 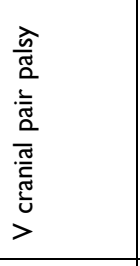 & 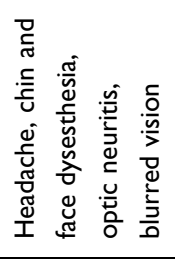 & 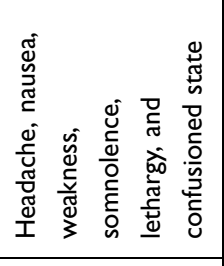 & 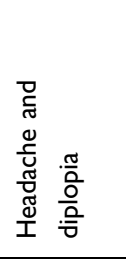 \\
\hline 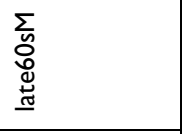 & $\stackrel{\infty}{\cap} \Sigma$ & $\stackrel{\infty}{\circ \Sigma}$ & กี $\Sigma$ & ஜ ч & $\neq \Sigma$ & $\bar{\infty} \Sigma$ & ذँ \\
\hline 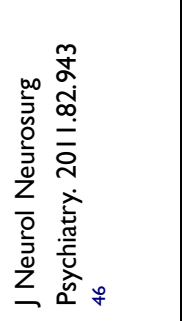 & 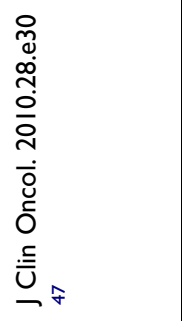 & 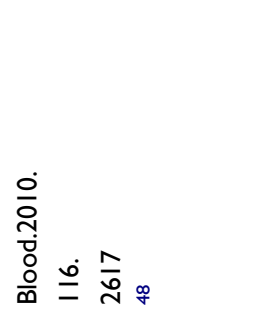 & 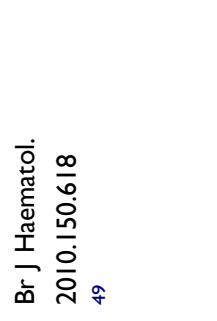 & 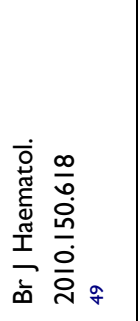 & 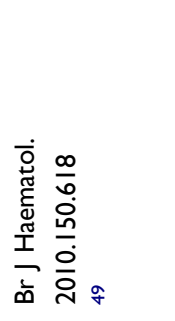 & 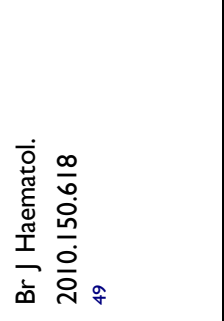 & 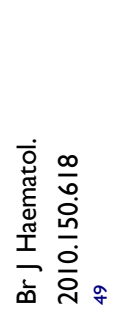 \\
\hline
\end{tabular}




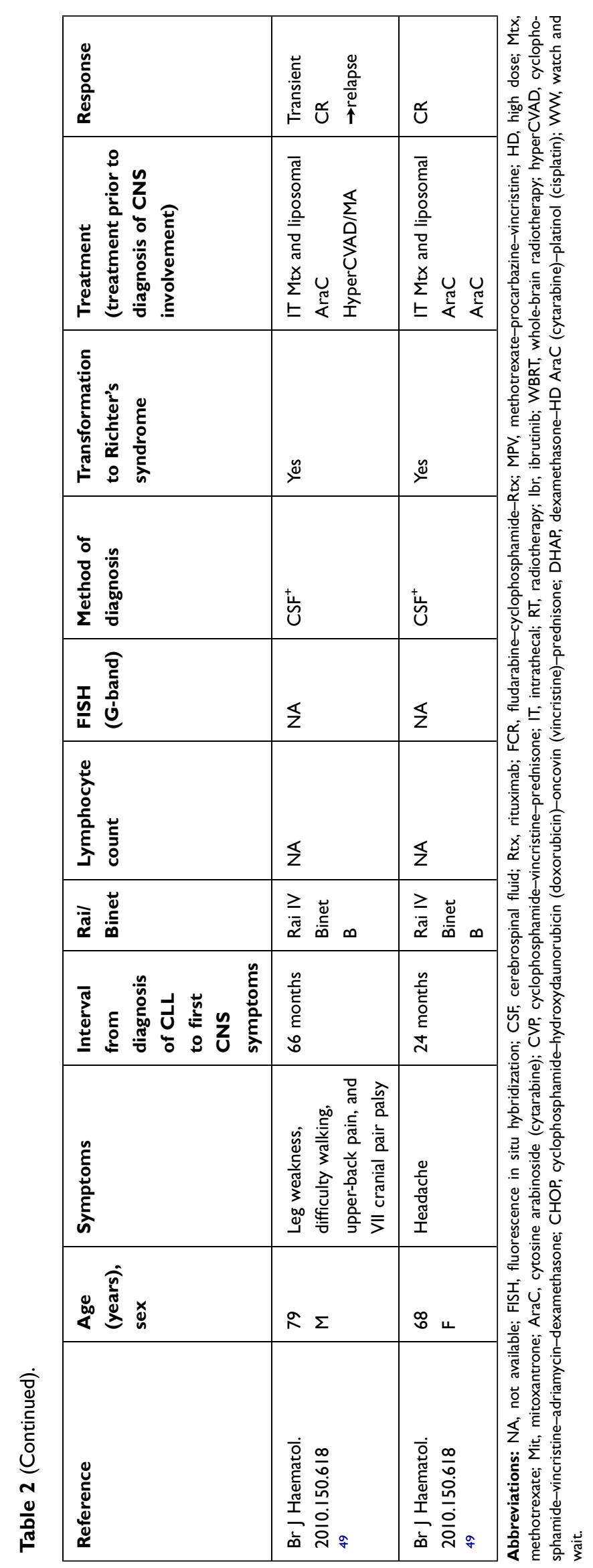


data from cases is important to verify the choice of new or combination drugs for administration from an early stage.

\section{Ethics}

Informed consent was provided by the patient on admission to have the case details published. The patient passed away before publication of causes not included in the case report. Institutional approval was not required for publication.

\section{Acknowledgments}

We thank Nicole Clarke, PhD from Edanz Group for editing a draft of this manuscript.

\section{Author Contributions}

All authors contributed to data analysis, drafting or revising the article, have agreed on the journal to which the article will be submitted, gave final approval to the version to be published, and agree to be accountable for all aspects of the work.

\section{Disclosure}

The authors report no conflicts of interest in this work.

\section{References}

1. Chihara D, Ito H, Matsuda T, et al. Differences in incidence and trends of haematological malignancies in Japan and the United States. $\mathrm{Br} J$ Haematol. 2014;164(4):536-545. doi:10.1111/bjh.12659

2. Hallek M. Chronic lymphocytic leukemia: 2020 update on diagnosis, risk stratification and treatment. Am J Hematol. 2019;94(11):12661287. doi:10.1002/ajh. 25595

3. Stilgenbauer S, Zenz T. Understanding and managing ultra high-risk chronic lymphocytic leukemia. Hematology Am Soc Hematol Educ Program. 2010;2010:481-488. doi:10.1182/asheducation-2010.1.481

4. Hanse MC, Van't Veer MB, van Lom K, et al. Incidence of central nervous system involvement in chronic lymphocytic leukemia and outcome to treatment. $J$ Neurol. 2008;255(6):828-830. doi:10.1007/ s00415-008-0710-4

5. Lopes da Silva R. Spectrum of neurologic complications in chronic lymphocytic leukemia. Clin Lymphoma Myeloma Leuk. 2012;12 (3):164-179. doi:10.1016/j.clml.2011.10.005

6. Bernard S, Goldwirt L, Amorim S, et al. Activity of ibrutinib in mantle cell lymphoma patients with central nervous system relapse. Blood. 2015;126(14):1695-1698. doi:10.1182/blood-2015-05-647834

7. Wanquet A, Birsen R, Bonnet C, et al. Management of central nervous system involvement in chronic lymphocytic leukaemia: a retrospective cohort of 30 patients. Br J Haematol. 2017;176(1):37-49. doi:10.1111/ bjh. 14387

8. Moazzam AA, Drappatz J, Kim RY, et al. Chronic lymphocytic leukemia with central nervous system involvement: report of two cases with a comprehensive literature review. J Neurooncol. 2012;106 (1):185-200. doi:10.1007/s11060-011-0636-z

9. Molica S. A systematic review on Richter syndrome: what is the published evidence? Leuk Lymphoma. 2010;51(3):415-421. doi:10.3109/ 10428190903515192
10. Cramer SC, Glaspy JA, Efird JT, et al. Chronic lymphocytic leukemia and the central nervous system: a clinical and pathological study. Neurology. 1996;46(1):19-25. doi:10.1212/WNL.46.1.19

11. Brick WG, Majmundar M, Hendricks LK, et al. Leukemic leptomeningeal involvement in stage 0 and stage 1 chronic lymphocytic leukemia. Leuk Lymphoma. 2002;43(1):199-201. doi:10.1080/10428190210191

12. Cabannes-Hamy A, Lemal R, Goldwirt L, et al. Efficacy of ibrutinib in the treatment of Bing-Neel syndrome. Am J Hematol. 2016;91(3): E17-19. doi:10.1002/ajh.24279

13. Tucker DL, Naylor G, Kruger A, et al. Ibrutinib is a safe and effective therapy for systemic mantle cell lymphoma with central nervous system involvement - a multi-centre case series from the United Kingdom. Br J Haematol. 2017;178(2):327-329. doi:10.1111/bjh.14122

14. Lionakis MS, Dunleavy K, Roschewski M, et al. Inhibition of B cell receptor signaling by ibrutinib in primary CNS lymphoma. Cancer Cell. 2017;31(6):833-843.e835. doi:10.1016/j.ccell.2017.04.012

15. Grommes C, Pastore A, Palaskas N, et al. Ibrutinib unmasks critical role of bruton tyrosine kinase in primary CNS lymphoma. Cancer Discov. 2017;7(9):1018-1029. doi:10.1158/2159-8290.CD-17-0613

16. Rizvi W, Truong Q. Unusual relapse of chronic lymphocytic leukemia after remission. Cureus. 2018;10(2):e2176.

17. Beziat G, Gauthier M, Protin C, et al. Venetoclax with high-dose methotrexate and rituximab seem effective and well-tolerated in the treatment of central nervous system involvement of chronic lymphocytic leukemia: a case report. Clin Case Rep. 2020;8(2):269-273. doi: $10.1002 / \mathrm{ccr} 3.2580$

18. Reda G, Cassin R, Dovrtelova G, et al. Venetoclax penetrates in cerebrospinal fluid and may be effective in chronic lymphocytic leukemia with central nervous system involvement. Haematologica. 2019;104(5):e222-e223. doi:10.3324/haematol.2018.213157

19. Akdogan O, Guven T, Altindal S, et al. An uncommon neurological manifestation of chronic lymphocytic leukemia: longitudinally extensive transverse myelitis. Mult Scler Relat Disord. 2020;37:101455. doi:10.1016/j.msard.2019.101455

20. Witton LA, Menon S, Perera KS. Central nervous system involvement with chronic lymphocytic leukemia. Can J Neurol Sci. 2019;46 (5):640-641. doi:10.1017/cjn.2019.75

21. Gallastegui N, Cassidy DP, Heros DO, et al. Central nervous system involvement by small lymphocytic lymphoma after a myxomarelated embolic event. Case Rep Hematol. 2019;2019:1825491.

22. Guo R, Zhang X, Niu C, et al. Primary central nervous system small lymphocytic lymphoma in the bilateral ventricles: two case reports. BMC Neurol. 2019;19(1):200. doi:10.1186/s12883-019-1430-3

23. Otani R, Uzuka T, Matsuda $\mathrm{H}$, et al. Brain invasion by chronic lymphocytic leukemia. Neuropathology. 2019;39(1):54-57. doi:10. 1111/neup. 12525

24. Mousinho F, Mendes T, Sousa E, et al. Treatment sequencing in a chronic lymphocytic leukemia patient with central nervous system involvement. Case Rep Hematol. 2018;2018:7817918.

25. Wąsik-Szczepanek E, Szymczyk A, Szczepanek D, et al. Richter syndrome: a rare complication of chronic lymphocytic leukemia or small lymphocytic lymphoma. Adv Clin Exp Med. 2018;27 (12):1683-1689. doi:10.17219/acem/75903

26. Christoforidou A, Kapsas G, Bezirgiannidou Z, et al. Successful treatment of chronic lymphocytic leukemia multifocal central nervous system involvement with ibrutinib. Turk J Haematol. 2018;35 (2):147-149. doi:10.4274/tjh.2017.0313

27. Timmers NKLM, de Maar JS, van Kruijsdijk RCM, et al. Central nervous system localisation of chronic lymphocytic leukaemia, description of two very distinct cases and a review of the literature. Ann Hematol. 2018;97(9):1627-1632. doi:10.1007/s00277-018-3329-2

28. Xu L, Song JC, Sun XH, et al. Richter's syndrome of the central nervous system diagnosed concurrently with chronic lymphocytic leukaemia: a case report and literature review. Medicine (Baltimore). 2018;97(41):e12701. doi:10.1097/MD.00000000000 12701 
29. Albakr A, Alhothali W, Samghabadi P, et al. Central nervous system lymphoma in a patient with chronic lymphocytic leukemia: a case report and literature review. Cureus. 2018;10(11):e3660.

30. Mihaljevic B, Smiljanic M, Antic D, et al. Chronic lymphocytic leukemia involvement of central nervous system: clinical diversity, diagnostic algorithm and therapeutic challenges. Ann Indian Acad Neurol. 2018;21(1):85-87.

31. Tam CS, Kimber T, Seymour JF. Ibrutinib monotherapy as effective treatment of central nervous system involvement by chronic lymphocytic leukaemia. Br J Haematol. 2017;176(5):829-831. doi:10.1111/ bjh. 14000

32. Rojas-Hernandez CM, Nemunaitis J, Marjon KD, et al. Chronic lymphocytic leukemia with clinical debut as neurological involvement: a rare phenomenon and the need for better predictive markers. BMC Hematol. 2017;17:3. doi:10.1186/s12878-017-0073-0

33. Khan K, Malik AI, Almarzouqi SJ, et al. Optic neuropathy due to chronic lymphocytic leukemia proven with optic nerve sheath biopsy. $J$ Neuroophthalmol. 2016;36(1):61-66. doi:10.1097/WNO.00000000000 00300

34. Wanquet A, Birsen R, Lemal R, et al. Ibrutinib responsive central nervous system involvement in chronic lymphocytic leukemia. Blood. 2016;127(19):2356-2358. doi:10.1182/blood-2016-02-697193

35. de Souza SL, Santiago F. Ribeiro-Carvalho, MeM, et al. Leptomeningeal involvement in B-cell chronic lymphocytic leukemia: a case report and review of the literature. BMC Res Notes. 2014;7::645.

36. Rossi C, Brisou G, Baseggio L, et al. Central nervous system involvement in chronic lymphocytic leukemia: uncommon manifestation with undefined therapeutic management. Leuk Lymphoma. 2014;55 (8):1939-1941. doi:10.3109/10428194.2013.858152

37. Aziz M, Chaurasia JK, Khan R, et al. Primary low-grade diffuse small lymphocytic lymphoma of the central nervous system. BMJ Case Rep. 2014;2014.

38. Benjamini O, Jain P, Schlette E, et al. Chronic lymphocytic leukemia with central nervous system involvement: a high-risk disease? Clin Lymphoma Myeloma Leuk. 2013;13(3):338-341. doi:10.1016/j. clm1.2012.12.007

39. Jain P, Benjamini O, Pei L, et al. Central nervous system Richter's transformation and parvovirus B19 infection. Leuk Lymphoma. 2013;54(9):2070-2072. doi:10.3109/10428194.2013.765565
40. Ishida F, Nakazawa H, Takezawa Y, et al. Richter transformation in the brain from chronic lymphocytic leukemia. J Clin Exp Hematop. 2013;53(2):157-160. doi:10.3960/jslrt.53.157

41. Gonsalves WI, Zent CS, Pulido JS, et al. Visual loss in early-stage chronic lymphocytic leukemia. J Clin Oncol. 2013;31(17):e280-282. doi:10.1200/JCO.2012.46.7431

42. Stuplich M, Mayer K, Kim Y, et al. Richter syndrome and brain involvement: low-grade lymphoma relapsing as cerebral high-grade lymphoma. Acta Haematol. 2012;127(2):93-95. doi:10.1159/ 000334068

43. Imitola J, Pitt K, Peoples JL, et al. Multifocal CNS infiltration of chronic lymphocytic leukemia in the form of small-cell solid metastatic lesions. J Neurooncol. 2012;109(1):213-215. doi:10.1007/ s11060-012-0869-5

44. Cohen JB, Cavaliere R, Byrd JC, et al. Hearing loss due to infiltration of the tympanic membrane by chronic lymphocytic leukemia. Case Rep Hematol. 2012;2012:589718.

45. Ackermann KA, Z'Graggen WJ, El-Koussy M, et al. Blindness in a patient with chronic lymphocytic leukemia. Am J Hematol. 2011;86 (9):783-784. doi:10.1002/ajh.22042

46. Graves TD, Collins GP, Parry A. Richter's syndrome of the brain and the spinal cord. J Neurol Neurosurg Psychiatry. 2011;82(8):943-944. doi:10.1136/jnnp.2010.209759

47. Kakimoto T, Nakazato T, Hayashi R, et al. Bilateral occipital lobe invasion in chronic lymphocytic leukemia. J Clin Oncol. 2010;28(3): e30-32. doi:10.1200/JCO.2009.23.8436

48. Russwurm G, Heinsch M, Radkowski R, et al. Dasatinib induces complete remission in a patient with primary cerebral involvement of B-cell chronic lymphocytic leukemia failing chemotherapy. Blood. 2010;116(14):2617-2618. doi:10.1182/blood-2010-04-279786

49. Calvo-Villas JM, Fernández JA, de la Fuente I, et al. Intrathecal liposomal cytarabine for treatment of leptomeningeal involvement in transformed (Richter's syndrome) and non-transformed B-cell chronic lymphocytic leukaemia in Spain: a report of seven cases. Br J Haematol. 2010;150(5):618-620. doi:10.1111/j.1365-2141. 2010.08238.x
Journal of Blood Medicine

\section{Publish your work in this journal}

The Journal of Blood Medicine is an international, peer-reviewed, open access, online journal publishing laboratory, experimental and clinical aspects of all aspect pertaining to blood based medicine including but not limited to: Transfusion Medicine; Blood collection, Donor issues, Transmittable diseases, and Blood banking logistics; Immunohematology; Artificial and alternative blood based

\section{Dovepress}

therapeutics; Hematology; Biotechnology/nanotechnology of blood related medicine; Legal aspects of blood medicine; Historical perspectives. The manuscript management system is completely online and includes a very quick and fair peer-review system. Visit http://www.dovepress.com/testimonials.php to read real quotes from published authors. 